\title{
Evaluation of MR/Fluoroscopy-guided Portosystemic Shunt Creation in a Swine Model
}

\author{
Aravind Arepally, MD, Parag V. Karmarkar, MS, Di Qian, MS, Brad Barnett, BS, and Ergin Atalar, PhD
}

PURPOSE: To evaluate three different percutaneous portosystemic shunts created with magnetic resonance (MR) imaging and fluoroscopy guidance in a swine model.

MATERIALS AND METHODS: In stage 1 of the experiment, an active MR intravascular needle system was created for needle tracking and extracaval punctures. Twenty inferior vena cava (IVC)/superior mesenteric vein (SMV)/portal vein $(\mathrm{PV})$ punctures were performed in 10 swine (weight, $40-45 \mathrm{~kg}$ ) in a 1.5-T short-bore interventional MR imager. With use of a real-time MR imaging sequence, the needle was guided through the IVC and into the SMV or PV $(N=$ 20 punctures). After confirmation, a wire was advanced into the portal venous system under MR imaging guidance $(N=20)$. In stage 2 , animals were transferred to the radiographic fluoroscopy suite for deployment of shunts. Three different shunts were evaluated in this study: (i) a commercial stent-graft, (ii) a prototype bridging stent, and (iii) a prototype nitinol vascular anastomotic device. Postprocedural necropsy was performed in all animals.

RESULTS: Successful MR-guided IVC/SMV punctures were performed in all 20 procedures (100\%). All three shunts were deployed. Stent-grafts had the poorest mechanism for securing a shunt. The vascular anastomotic device and the bridging stent had more secure anchoring mechanisms but also had higher technical failure rates $(50 \%$ and $40 \%$, respectively). When deployed successfully, the vascular anastomotic device resulted in no bleeding at the sites of punctures at necropsy.

CONCLUSION: Percutaneous shunts and vascular anastomoses between the portal mesenteric venous system and IVC were successfully created with use of a combination of MR imaging and conventional fluoroscopy for guidance.

J Vasc Interv Radiol 2006; 17:1165-1173

Abbreviations: IVC = inferior vena cava, $\mathrm{PV}=$ portal vein, $\mathrm{SMV}=$ superior mesenteric vein, TIPS = transjugular intrahepatic portosystemic shunt

DESPITE advances in medical and surgical therapies, the management of portal hypertension remains a challenge. Current options include surgical creation of a portosystemic shunt, liver transplantation, and creation of a

\footnotetext{
From the Russell H. Morgan Department of Radiology and Radiological Science (A.A., P.V.K., D.Q., B.B., E.A.), The Johns Hopkins Medical Institutions, Blalock 545, 600 North Wolfe Street, Baltimore, Maryland 21287; and Department of Electrical Engineering (E.A.), Bilkent University, Ankara, Turkey. Received December 1, 2005; revision requested March 6; final revision received and accepted May 3, 2006. Address correspondence to A.A; E-mail: aarepal@jhmi.edu
}

This study was supported in part by National Institutes of Health grant 1 K08 EB004348-01, R01 HL61672. None of the authors have identified a conflict of interest.

(c) SIR, 2006

DOI: 10.1097/01.RVI.0000228493.07075.FC transjugular intrahepatic portosystemic shunt (TIPS) (1). As a result of the limited availability of donor livers and technical surgical expertise, transplantation is not a viable option for the majority of candidates (2).

Surgical portosystemic shunts have traditionally been accomplished with (i) a surgical end-to-side mesocaval shunt that decompresses the portal vein (PV) or (ii) a side-to-side splenorenal shunt to decompress the splenic vein $(3,4)$. Because the creation of these shunts is selective, a significant portion of portal venous flow is maintained, and these shunts are therefore associated with a low rate of encephalopathy and recurrent bleeding (5). However, these surgical procedures have also been associated with high complication rates. Particularly in patients with extensive retroperitoneal collateral vessels, surgical interventions can be difficult, and perioperative mortality rates of $20 \%-50 \%$ have been noted (6). By contrast, creation of a TIPS is associated with higher incidences of encephalopathy, recurrent bleeding, and shunt occlusion (4) compared with surgical shunts. In addition, because the procedure is performed without direct visualization of the portal venous system, potential complications include traversal of the liver capsule and creation of fistulous tracts from the shunt to the hepatic artery or bile ducts (7). However, the percutaneous creation of a portomesenteric/caval shunt may provide the maximum advantage of a selective shunt with the benefit of lower complication rates.

The goals of this study were to demonstrate the feasibility of creating 


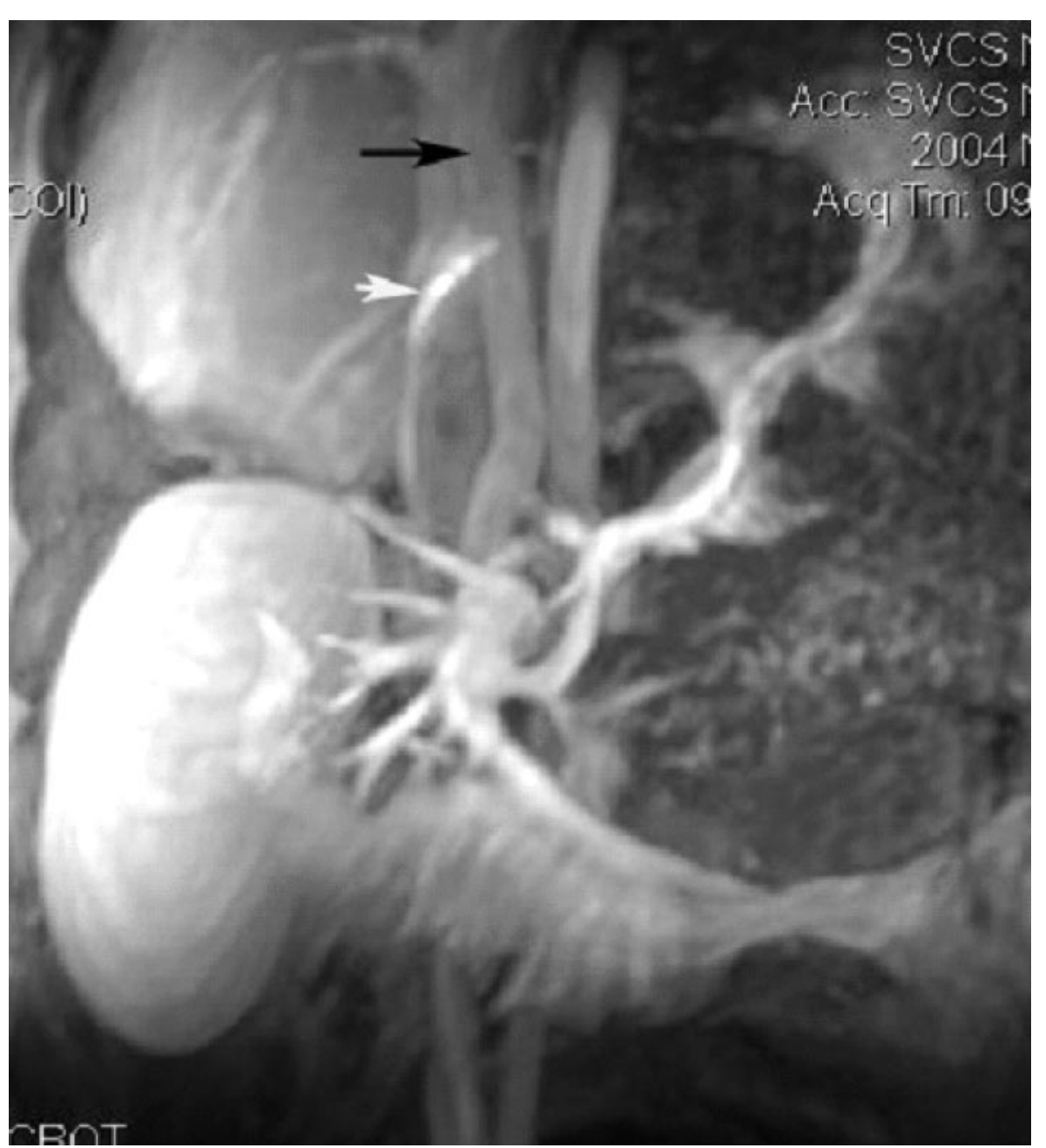

Figure 1. Conventional MR angiography/venography of the mesenteric venous system performed with $30 \mathrm{~mL}$ of gadolinium diethylenetriamine pentaacetic acid (4.8-msec repetition time; $1.4-\mathrm{msec}$ echo time; $25^{\circ}$ flip angle; $256 \times 256$ image matrix) before any punctures. White arrow indicates active needle; black arrow indicates PV.

a percutaneous extrahepatic portomesenteric shunt under guidance by magnetic resonance (MR) imaging/ conventional fluoroscopy and to assess the three types of shunts created.

\section{MATERIALS AND METHODS}

\section{Animal Model}

The institutional animal care and use committee approved the animal studies. We performed experiments on 10 healthy swine (weight, 40-45 $\mathrm{kg}$ ). Sedation was achieved with xylazine and ketamine. After endotracheal intubation, inhaled isoflurane (2\%) was provided during mechanical ventilation with oxygen (98\%). Percutaneous access into the right femoral vein was achieved under ultrasound (US) guidance, followed by placement of a 12-F sheath into the femoral vein. All animals were transferred to the MR suite for the remaining portion of the procedure.

\section{Needle Design}

The needle design of the active MR needle, the prototype of which was developed in our laboratory (by P.K. and E.A.), has been previously described (8). This needle is made of two concentrically configured pieces of nitinol tubing that are insulated from each other, with the inner tubing extending $4 \mathrm{~cm}$ beyond the outer tubing to form a loopless antenna (9). The intravascular needle antenna has a central lumen that can accommodate a 0.038-inch guide wire, and therefore the antenna can be safely advanced over the wire through a femoral vein approach. The needle system is $9 \mathrm{~F}$ in caliber with a sharpened three-face bevel at the distal end. To facilitate puncture, the shaft is preshaped at the distal tip to provide a $55^{\circ}$ angle for all vascular punctures. The inner lumen of the inner tube is further insulated with a liner that electrically isolates the system and acts as a guide wire lumen (0.038-inch wire compatible). The entire assembly is insulated with nylon to isolate the needle components from direct electrical contact with biologic fluids except at the distal tip of the needle. The radiofrequency circuitry matches the transmission at 63.86 $\mathrm{MHz}$ to enable active visualization of the needle (10) (Figs 1, 2). To perform transvascular punctures, the distal tip of the needle is oriented in the direction of the puncture. A second standard nitinol guide wire (ev3, Plymouth, MN) has been modified so that the tip (0.035 inches) is sharpened and is advanced $1 \mathrm{~cm}$ outside the active needle to facilitate the puncture.

\section{Shunt Design}

One commercial shunt (shunt A) and two custom-manufactured shunts (shunts B and C) shunts were evaluated in the study.

Shunt A.-Shunt A was a commercially available covered stent-graft (Viabahn; W.L. Gore \& Associates, Flagstaff, AZ). This stent-graft is a flexible, self-expanding stent that consists of an expanded polytetrafluoroethylene lining with an external nitinol support extending along the entire length. The stent-graft is attached to a dual-lumen polyethylene delivery catheter, and the device used in this study had a working length of $75 \mathrm{~cm}$. The delivery catheter is attached to a three-port clear plastic adapter (ie, hub assembly) that includes a central port for guide wire introduction, a second port for system flushing, and a third port for the deployment system. Five different stent grafts $(8 \mathrm{~mm}$ in diameter, $n$ $=2 ; 10 \mathrm{~mm}$ in diameter and $6 \mathrm{~cm}$ in length, $n=3$ ) were used for this study.

Shunt B.-Shunt B (Fig 3) was constructed in our laboratory and was 

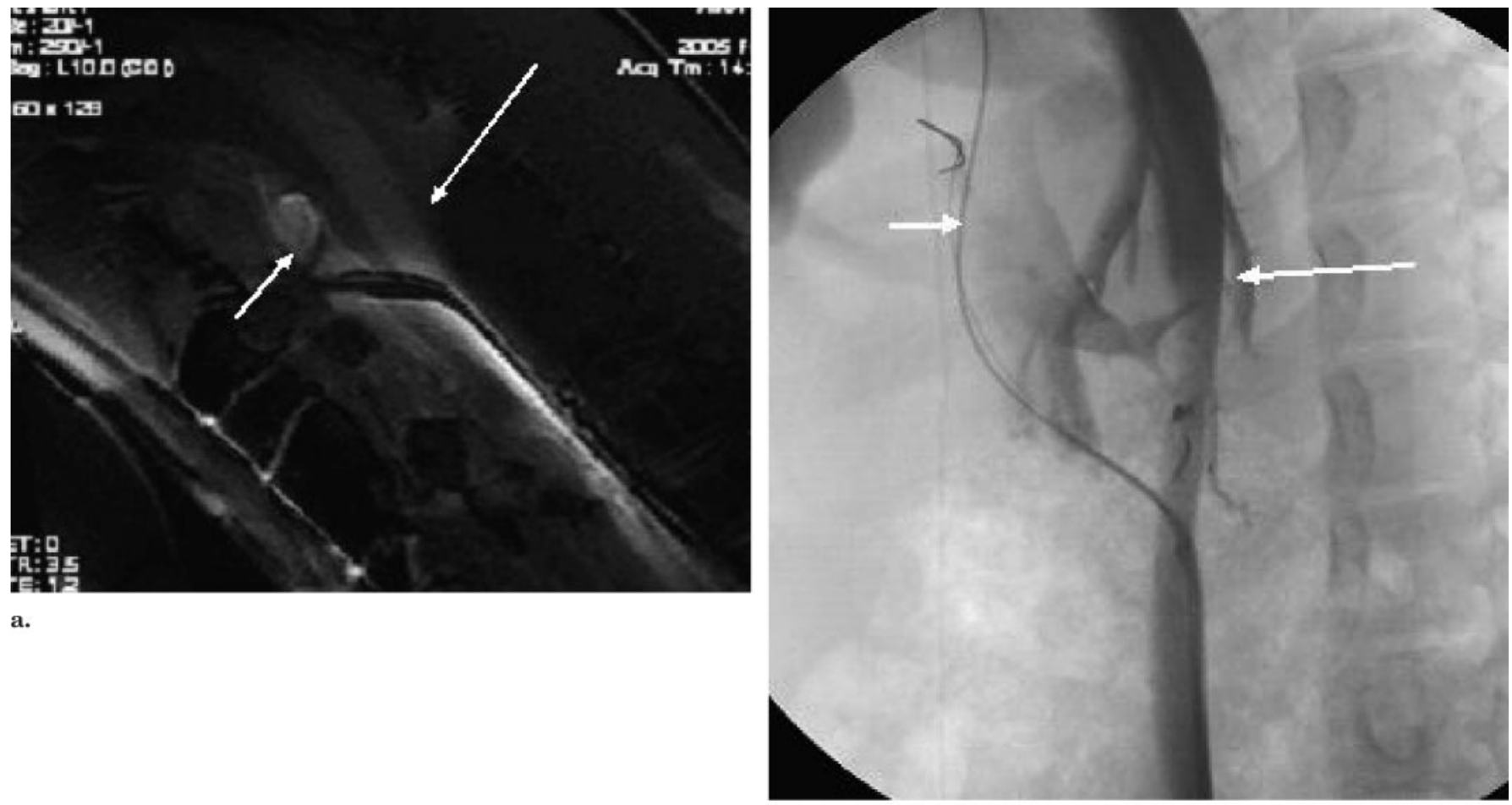

b.

Figure 2. (a) MR-guided puncture and catheterization of the mesenteric venous circulation performed with real-time gradient-recalled echo sequence (3.4-msec repetition time; 1.2 -msec echo time; $45^{\circ}$ flip angle; 30-cm field of view; $6-8$ frames/sec) in combination with an interactive scan plane acquisition. Short arrow indicates PV; long arrow indicates IVC. (b) Conventional venogram of PV (short arrow) and IVC (long arrow) before deployment of shunts.

designed to serve as a bridging stent between the two vascular beds. The centerpiece of the shunt device was made of a section of a nitinol stent $1 \mathrm{~cm}$ in diameter and $1 \mathrm{~cm}$ long. Three nitinol anchoring struts were vertically attached symmetrically around the stent body with $120^{\circ}$ of radial distance from each other. Each end of the nitinol strut had a $1-\mathrm{cm}$ long flange protruding outward from the stent. These flanges provided an anchoring force for the shunt device so that when the device was deployed, the flanges on each end hooked onto the inner wall of each vessel and locked the two vessels together. The bridging stent was deployed with use of a stent delivery catheter, which deployed the bridging stent in two stages. To create the shunt, the distal anchors of this device were first deployed in the portal/mesenteric system. The deployed anchors were then used to pull the portal/mesenteric veins closer to the IVC so that the proximal anchors in the IVC could be released and deployed.

Shunt C.- Shunt C (Fig 4) was constructed in our laboratory and was designed to serve as an anastomosis between the two vascular beds. The anastomotic device was made of a single piece of 0.009-inch nitinol shapememory wire (NDC, Fremont, CA). The nitinol wire was bent into a fixed shape and mounted on a stainless-steel frame. This preshaped nitinol wire was then placed inside a high-power electrical furnace and heated for $20 \mathrm{~min}$ utes at $550^{\circ} \mathrm{C}$. The nitinol wire would then maintain this shape at room temperature. The shape of the anastomotic device was a short-bore ring with two sets of flanges on each side. The diameter of the ring was approximately $1 \mathrm{~cm}$ when fully relaxed, and the length was approximately $3 \mathrm{~mm}$. There were six flanges on each side of the ring, and the flange was $5 \mathrm{~mm}$ long. The flanges were placed symmetrically around the ring with a radial distance of $30^{\circ}$ from each other.
The anastomotic device was placed in the same way as the shunt device inside a delivery catheter and was deployed with the same technique. The shortened device body held the two vessels close to each other and therefore minimized blood leakage from the vessels.

\section{Technique}

Creation of percutaneous portosystemic shunts was performed with use of interventional MR imaging (stage 1) and conventional fluoroscopy (stage 2).

Stage 1: MR-guided access to portalmesenteric venous system.-Thirteen IVC/superior mesenteric vein (SMV)/ PV punctures were performed in 10 swine in a 1.5-T short-bore interventional MR scanner (CV/i; GE Medical Systems, Milwaukee, WI). Images were acquired by a combination of external phased-array coils and an intravascular needle. The needle was introduced from the common femo- 


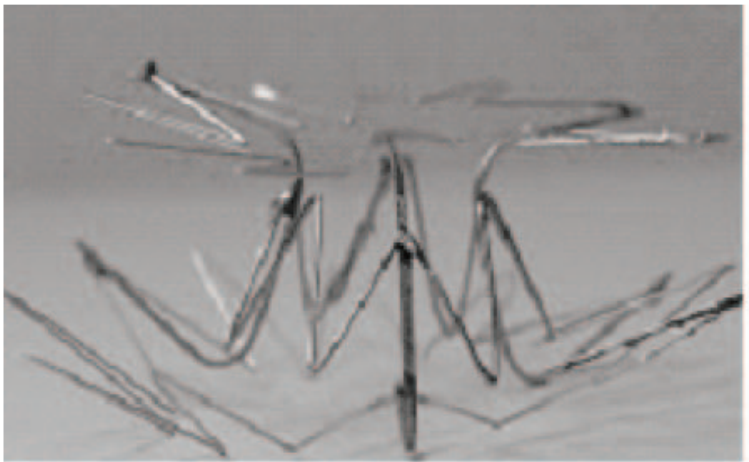

a.

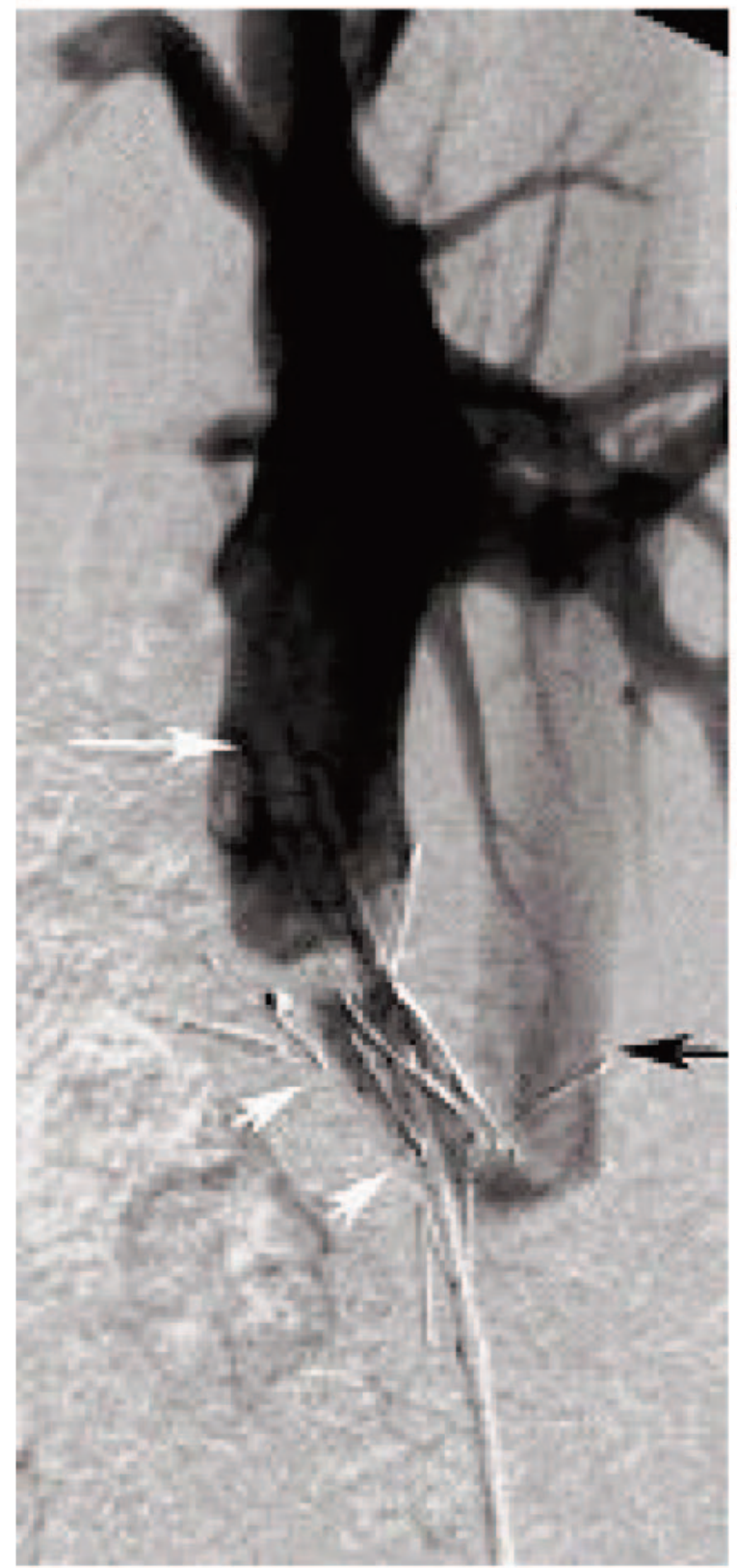

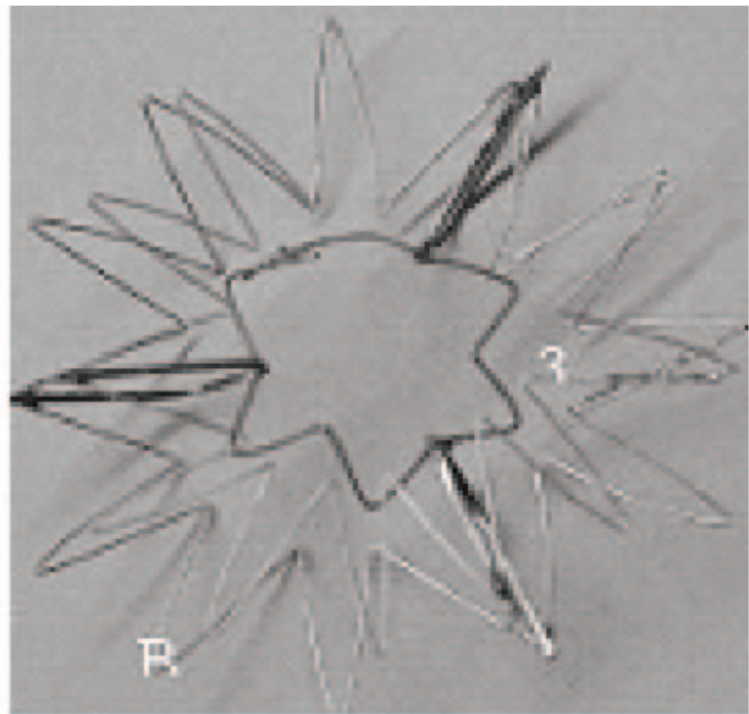

b.

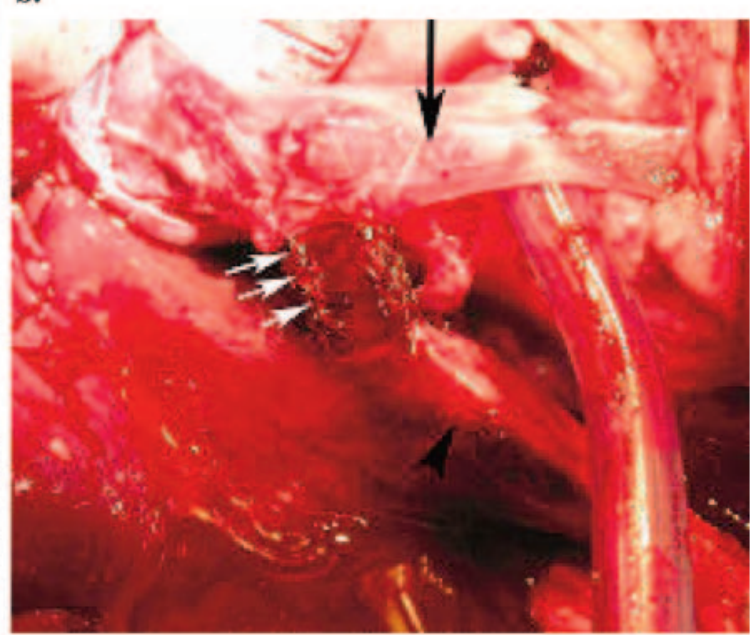

d.

Figure 3. (a,b) Lateral and anterior views of shunt $\mathrm{B}$. The centerpiece of the shunt device is made from a section of a nitinol stent $1 \mathrm{~cm}$ in diameter and $1 \mathrm{~cm}$ long. Three nitinol anchoring struts are vertically attached symmetrically around the stent body, with $120^{\circ}$ of radial distance from each other. Each end of the nitinol strut has a 1-cm-long flange protruding outward from the stent. (c) Venogram after deployment of shunt B (white arrowheads) from the PV (short white arrow) to the IVC (black arrow). There is no evidence of extravasation at angiography. (d) At necropsy, shunt (white arrowheads) is intact and bridges both vascular beds. Long black arrow indicates PV; black arrowhead indicates IVC.

c. 


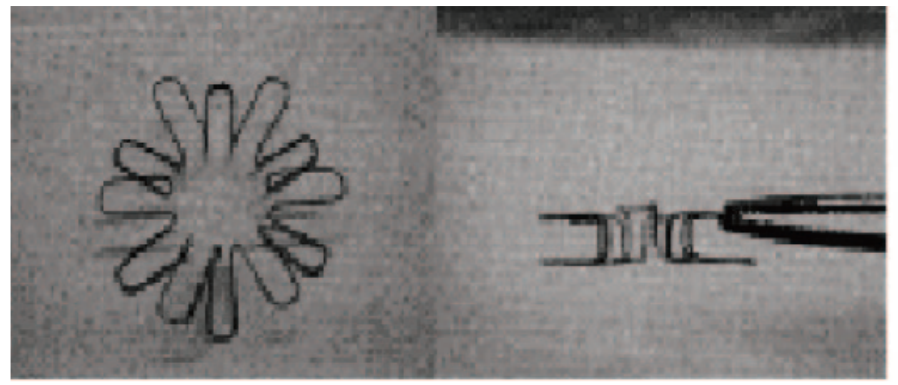

a.

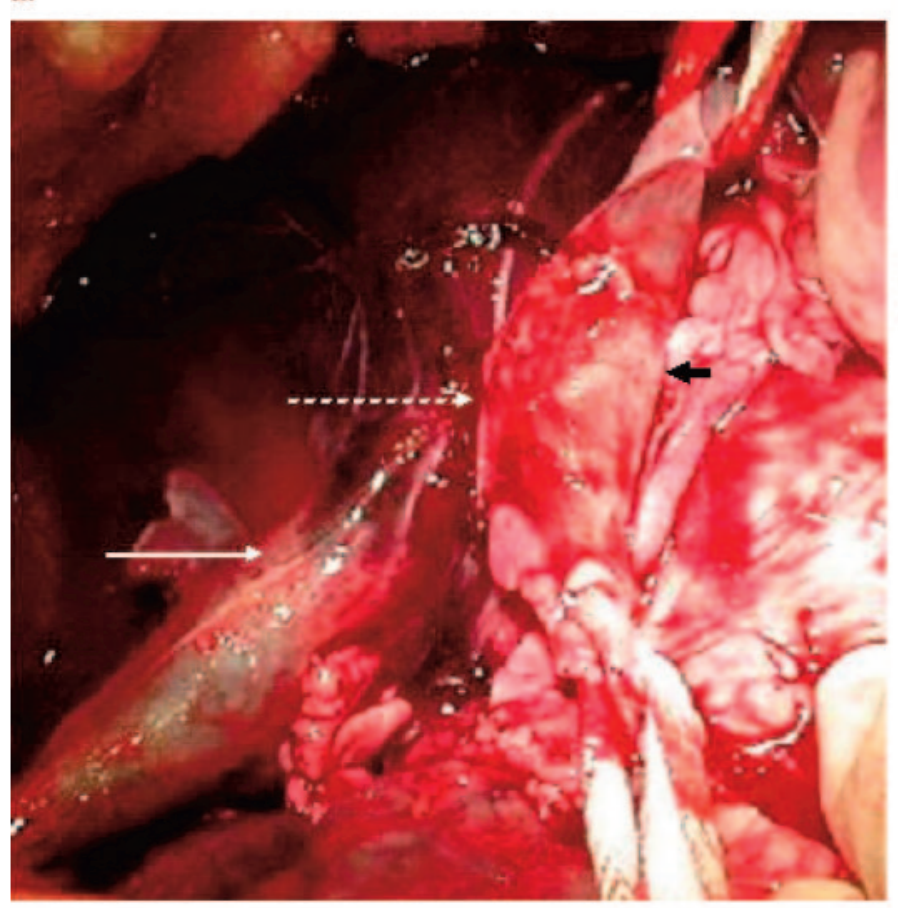

f.

ral vein through a standard $12-\mathrm{F}$ vascular sheath and advanced over a 0.035-inch nitinol guide wire (ev3).

All procedures were performed by an experienced interventional radiologist. During the procedure, the interventionalist advanced the needle with use of an imaging console adjacent to the MR imaging scanner to monitor needle tracking and orientation. A separate person controlled the imaging parameters and slice orientation based on the feedback of the interventionalist. The needle was positioned in the IVC and rotated to the correct orientation according to a real-time gradient-recalled echo sequence. The needle was readily tracked at all times, and multiple projections were used to confirm the needle position throughout the procedure. After the needle was introduced and before performance of any puncture, MR angiography/venography of the mesenteric venous system (Fig 1) was performed with $30 \mathrm{~mL}$ of gadolinium diethylenetriamine pentaacetic acid (4.8-msec repetition time; $1.4-$ msec echo time; $25^{\circ}$ flip angle; $31.2-\mathrm{kHz}$ bandwidth; field of view, $256 \times 256$ image matrix)

With a real-time gradient-recalled echo sequence (3.4-msec repetition time; $1.2-\mathrm{msec}$ echo time; $45^{\circ}$ flip angle; 30-cm field of view; 6-8 frames/ sec), in combination with an interactive scan plane acquisition (i-Drive; GE Medical Systems), the needle was advanced into the IVC and guided to the level where the SMV was closest to the IVC (Fig 3a, b). After proper orientation of the active needle toward the target vessel, the path of the needle was examined to confirm that no vessels or retroperitoneal structures would be inadvertently injured. Next, a second standard nitinol guide wire with a sharpened tip (ev3) was coaxially introduced. The sharpened tip was advanced $1 \mathrm{~cm}$ outside the active needle to facilitate the puncture; passive tracking of the sharpened tip was used to monitor the progress of the needle as it exited the IVC and entered the SMV. Under a real-time gradientrecalled echo sequence with multiplanar views, the entire system (ie, sharpened needle and active needle) was advanced as a unit until the PV/SMV was entered. After removal of the sharpened guide wire, immediate return of blood through the active needle confirmed that the SMV had been successfully punctured. After removal of the guide wire, a direct MR portogram was obtained through the needle with use of $10 \mathrm{~mL}$ of gadolinium di- 
ethylenetriamine pentaacetic acid at a concentration of $25 \%$ (fast spoiled gradient echo sequence; 6-msec repetition time; $1.3-\mathrm{msec}$ echo time; $90^{\circ}$ flip angle; no slice selection; excitations, 0.5 ; $45-\mathrm{cm} \times 22.5-\mathrm{cm}$ field of view; 3 frames/sec). In three animals (when allotted MR imaging time allowed), two separate punctures were made. After confirmation, a wire (018 inches or 0.035 inches) was advanced into the portal venous system under MR imaging guidance $(n=13)$ (Fig 2).

Stage 2: creation of portal/mesocaval shunt.-After successful access into the portomesenteric venous circulation, animals were transferred to a conventional fluoroscopic suite for deployment of shunts. Under fluoroscopic visualization, the puncture needle was removed and angioplasty of the retroperitoneum was performed with a 6-mm $\times$ 4-cm balloon (Cordis, Miami, FL). After angioplasty, devices were then advanced to create the shunt.

Shunt A was deployed in a standard fashion as described by the manufacturer. To launch shunts B and C, a device-deploying catheter was designed specifically for this purpose, similar to the commercial sheathbased delivery systems used for selfexpanding stents. The delivery system was compatible with a 10-F, 0.038inch wire. The delivery system was made of two components, the inner tubing assembly and the outer delivery sheath. Shunts A and B were collapsed between the two and then released in stages by gradually pulling back on the sheath. The inner member was made of a nylon tube $80 \mathrm{~cm}$ long with a 0.038-inch wire-compatible central lumen. The proximal $76 \mathrm{~cm}$ of the tube was stiffened by the addition of a concentric stiff polyamide tube over the nylon tube. At the proximal end of the tube was a connector/hub, and at the distal end of the inner member was a cone-shaped nylon component to enable gradual advancement of the device into the PV through the vascular puncture. A stent pusher, which was a 3-mm-diameter tube, was glued $2 \mathrm{~cm}$ from the proximal end of the distal cone. The inner member was advanced in a $70-\mathrm{cm} 10-\mathrm{F}$ sheath (Fast Cath sheath; St. Jude Medical, Minnetonka, MN). The shunts (A and B) were collapsed on the inner member between the proximal end of the distal cone and the distal end of the stent pusher. This delivery system enabled easy advancement from one vessel to another as the device entered the punctured opening of the PV, as well as the ability to deliver the shunts in two stages. When the shunt device was deployed, the distal tip of the delivery catheter first entered the PV. Withdrawing the outer sheath by $1 \mathrm{~cm}$ released the distal anchoring flanges/vertical struts of the shunt device. With the flanges hooked to the inner wall of the PV, the physician could pull the vessel toward the IVC. Therefore, as the proximal section of the shunt device reentered the IVC, the outer sheath was then further withdrawn to release the second set of flanges, which locked onto the inner wall of the IVC. In this fashion, the shunt device was completely deployed; the nitinol stent tubing at the center would open and allow blood flow from the IVC to the PV.

After deployment of all shunts, catheter-based angiography was performed with a multiple-side hole straight catheter (Cook, Bloomington, IN) to assess the anastomosis for leakage and positioning of the grafts. Postprocedural necropsy was performed in all animals to assess for bleeding, and direct inspection of the anchoring mechanism of all shunts was performed.

\section{Data Analysis}

For stage 1 of the experiment, technical success for transcaval puncture was defined as traversal of the needle from the IVC to the SMV or PV without traversal of any retroperitoneal organs. In addition to success rates, the number of passes required for each successful procedure was noted. For stage 2 of the experiment, technical success for MR-guided shunt creation was based on angiography and direct inspection of each shunt. This was defined by the ability to bridge the two vascular beds with the shunt without traversing any organs. In addition, the stability of the anchoring mechanism of each shunt was assessed. When there was a successful creation of a shunt, bleeding at the site of the shunt was classified as (i) major if diffuse hemorrhage was present in the peritoneal cavity, (ii) moderate if bleeding and hemorrhage was localized to the shunt, or (iii) none if bleeding or hematoma could not be identified at the site of the shunt.

\section{RESULTS}

\section{Stage 1: MR-guided Access to Portal-mesenteric Venous System}

Successful MR-guided IVC/SMV/ PV punctures were performed in all 13 procedures (100\%). All procedures were performed with real-time MR imaging sequences with use of freebreathing techniques and without electrocardiographic gating. Punctures were made with no change in cardiac rhythm or rate and with no sequelae. As a result of the mobility of the SMV and PV, real-time imaging was necessary in all punctures to reorient the needle toward the target vessel. During real-time gradient-recalled echo sequences, 4-8 frames/sec were possible throughout the procedure. Active tracking of the needle as it traversed the IVC toward the SMV/PV was possible. All procedures were successful $(100 \%)$, with direct puncture of the SMV $(n=5)$ or PV $(n=8)$ and no traversal of any retroperitoneal organs/vessels. In 12 of 13 animals, punctures were made with one pass. One procedure took two passes to access the PV. On the basis of imaging and direct visualization, all punctures went directly into the target vessels without traversal of any retroperitoneal organs/vessels (Figs 1, 2).

\section{Stage 2: Creation of Portal/Mesocaval Shunt}

Shunt A.-With shunt A, the technical success rate was $100 \%$ with stent-grafts. However, on direct inspection, these stents also had the poorest mechanism for securing a shunt. Because of the trajectory of the needle punctures, the stents were unable to expand against the vascular lumen to create a stable shunt between the two vascular beds. As a result of this poor anchoring mechanism, a major amount of bleeding was noted around the shunt.

Shunt B.-With shunt B, the technical success rate was $60 \%$ (three of five cases). Failures were caused by malpositioning of the proximal anchors, which resulted in deployment into the retroperitoneum and not in the IVC. As a result of poor visibility of the anterior wall of the IVC, precise placement of the proximal anchors in the IVC was difficult. When the shunt was placed correctly, a 
moderate amount of bleeding around the shunt was noted in two of three swine. Angiography showed no leakage into the retroperitoneum.

Shunt C.-With shunt C, the vascular anastomotic device had the highest technical failure rate $(50 \%)$ but also had the most secure anchoring mechanism. The vascular anastomotic device allowed very close apposition of the vascular beds so any potential space was eliminated between the vascular lumens. There was no bleeding at the sites of the punctures at necropsy.

\section{DISCUSSION}

The creation of a percutaneous extrahepatic portosystemic shunt is contingent on two critical steps: (i) safe and dependable extrahepatic transcaval punctures into the portal circulation and (ii) a reliable conduit that will enable shunting into the systemic circulation. We demonstrated that with the use of multiplanar realtime MR imaging and conventional fluoroscopy, a percutaneous extrahepatic portosystemic shunt and an anastomosis can be constructed in a staged fashion. Under complete MR guidance, we were able to perform methodical and targeted access into the mesenteric/portal system. In addition, using this access, we were then able not only to create a conventional extrahepatic portosystemic shunt but also to then create a percutaneous anastomosis between the vessels.

\section{Extrahepatic Puncture of the Portomesenteric Venous System}

Because of the anatomic isolation of the mesenteric venous system, various authors have described percutaneous extrahepatic portal system shunt procedures (11-14). One limiting step in the evolution of this procedure has been a reliable technique to target and puncture the portal/mesenteric veins percutaneously. Initial attempts used fluoroscopy and dual catheters in the PV and IVC for complex fluoroscopic angulations and punctures. Kaminou et al (11) first described the use of this technique to puncture the splenic vein from the IVC. In a swine model, the PV was directly punctured, followed by placement of a wire basket into the splenic vein to serve as a target for the punctures. This procedure was successful in four of five swine in creating a shunt. However, in all four successful punctures, necropsy demonstrated that the needle and stent were placed through the pancreas. In addition to being fairly cumbersome, the inability to visualize retroperitoneal structures limited the utility of this technique.

In a study by Vivas et al (12), creation of an extrahepatic portacaval shunt in a canine model was performed with use of the jejunal veins from the mesentery that were exposed after a laparotomy. After placement of another catheter in the IVC, a blind puncture was made from the PV into the IVC. With this access, a covered prosthesis was placed to simulate a portacaval shunt. However, because of the lack of visibility, six of 10 animals died after the procedure as a result of major retroperitoneal bleeding caused by multiple vascular punctures. Both these studies further reinforce the importance of visualization of the retroperitoneum during these invasive procedures.

More recent developments have used adjunctive imaging to facilitate safe punctures. In studies performed by Wallace et al $(13,14)$, intravascular US was used for real-time image guidance. This allowed for a mean of 1.75 needle passes (range, 1-4) to enter the PV. However, intravascular US provides limited single axial plane imaging, and the target vein must be very close to the IVC for complete visualization to facilitate safe needle passage. These inherent limitations restrict the vasculature available for effective shunting to target vessels that are adjacent to the IVC.

During the past decade, MR guidance has been investigated as an alternate guidance modality for interventional procedures. In addition to high spatial resolution, MR imaging can provide real-time multiplanar imaging of the vascular system. In addition, the lack of ionizing radiation and/or iodinated contrast medium makes this an inviting modality for image-guided procedures. Kee et al $(15,16)$ initially described the use of MR imaging to replicate a TIPS procedure in an MR suite in animal and clinical studies. By use of a hybrid radiography/MR imaging suite and a modified nitinol TIPS needle set, MR-guided punctures into the intrahepatic PV were per- formed to replicate a TIPS procedure. For all the studies (16), the susceptibility artifact of the needle system was used to track the advancement and punctures (ie, passive tracking). However, passive tracking can be imprecise as a result of distortion of the imaging field and can be inaccurate in locating the catheter or needle.

As previously described, we have developed a completely active (ie, visible) needle that can be used for various endovascular interventions in the MR imaging suite (16). Unlike passive tracking, active tracking allows for precise localization and full visualization from the needle tip through the entire shaft. With the dual capability of real-time MR imaging and an active transvascular needle, we were able to take advantage of the three-dimensional anatomic vascular imaging provided by MR imaging to precisely monitor the needle throughout the entire puncture. With this dual capability, we could then evaluate the entire systemic, mesenteric, and portal circulation with standard MR angiographic imaging. This provided a roadmap for determining the optimal sites for IVC puncture and for entering the portal/ mesenteric circulation. In addition, in a real-time setting, we were able to calculate the angle between the target planes, the distance between the two vessels, and any displacement of the target vein during the punctures. Finally, the field of view could be readily adapted to monitor adjacent organs and vital vessels in a real-time setting. Because of these technical improvements, we believe MR guidance with an active transvascular needle is a safe and reliable technique for this stage of the procedure.

\section{Percutaneous Extrahepatic Portosystemic Shunts}

For clinical implementation, not only are safe and reliable transvascular punctures required, but an effective and stable shunt must be constructed between the two circulatory beds. The standard surgical mesocaval shunt is created by constructing a conduit between the SMV and the IVC (5). By contrast, a splenorenal shunt is simply constructed by creation of an anastomosis between the two veins (17).

The ideal percutaneous shunt would 
be able to simulate a surgical anastomosis. However, to our knowledge, no studies to date have been able to construct such a shunt; in fact, all previous animal studies have replicated surgical shunts by deploying a variety of percutaneous bridging stents to construct an extrahepatic portacaval shunt. Peterson et al (18) initially described the creation of a portacaval shunt with polytetrafluoroethylene-covered stent-grafts in an animal study and a very limited clinical trial. However, these studies involved only intrahepatic shunts that were similar to TIPS as a result of the intrahepatic location of the shunt. Wallace et al $(13,14)$ demonstrated an extrahepatic shunt in a feasibility and survival animal model using intravascular US and a flanged stent-graft. The initial feasibility study with their prototype design demonstrated that this type of shunt could be created, but the shunt had very poor patency, with near-complete occlusion of all stent-grafts placed. A study with longer follow-up (13), which used a newer stent design and adjunctive embolization of the PV to simulate portal hypertension, showed some improvement in patency, but five of six animals had $50 \%-100 \%$ narrowing at 12 -week follow-up. Finally, a recent study by Niyyati et al (19) described the use of intravascular US and a self-expanding stent with a submucosal covering to create a portacaval shunt. In findings similar to those of Wallace et al $(13,14)$, nearly all the animals had severe stenosis or rapid occlusion as a result of neointimal hyperplasia.

Despite the technical feasibility of these procedures, enthusiasm has been dampened, largely because of poor patency rates. This is primarily a result of the accelerated development of intimal hyperplasia seen with stent or stent-graft placement in the venous circulatory system. To improve patency, the hyperplasia should be addressed with some form of adjunctive pharmacologic therapy or by minimizing the physical length and dimensions of the shunts. In their feasibility study of portacaval shunts, Seong et al (20) commented that an ideal system would be a short stent-graft with a nitinol frame and a horizontal course with the capability to bring the veins together. Therefore, attempts to create a surgical anastomosis may provide the optimal alternative.

In our study, we have demon- strated that a variety of extrahepatic PV-to-IVC shunts can be created under guidance by MR imaging. We were able to construct these shunts from a commercially available stentgraft (shunt A) and a bridging nitinol stent (shunt B), and also to create a shunt with a vascular anastomosis (shunt C). Shunts A and B, which had very poor patency at follow-up, were similar in design to those used in earlier animal studies performed by several authors. Although the anchoring mechanism in shunt $B$ was more secure, it was inherently limited by the stent design, which has been shown to cause shunt thrombosis and intimal hyperplasia. However, in comparing the three shunts that were constructed, shunt $C$ is the design that merits further exploration. Although shunt $\mathrm{C}$ (ie, the anastomotic device) was associated with the highest technical failure rate $(50 \%)$, it also had the most secure anchoring mechanism. The vascular anastomotic device allowed very close apposition of the vascular beds so that any potential space was eliminated between the vascular lumens. This resulted in almost no bleeding at the sites of the punctures and demonstrated a complete vascular seal at necropsy.

MR guidance also allowed for full evaluation of the venous circulatory bed and the ability to create a trajectory into a desired vein that would be appropriate for anastomosis. In fact, as a result of the needle trajectory course, the stability of shunt A was weak, because the stents were unable to expand sufficiently against the vascular lumen. However, because of the horizontal course of the needle, the anastomosis device, which is composed of nitinol, was capable of pulling the target vein close to the IVC to create a vascular seal. When correctly deployed, the anastomosis allowed for very close apposition of the vascular beds. This provided the optimal shunt because any potential space was eliminated between the vascular lumens and there was no bleeding at the sites of punctures. In addition, because minimal nitinol material was left across the vascular lumens, this could minimize turbulence, which could affect patency rates. We also noted that, when the anastomosis is constructed safely, this was associated with almost no bleeding as well as a stable and secure seal between the two vascular beds.

Our main limitation in this study was the reliable construction of the anastomosis. Because of inherent problems with conventional fluoroscopy, there was poor visualization of the anterior IVC wall, which resulted in imprecise placement of the proximal phalanges of the anastomosis device. To address this problem, a potential solution will be to construct the entire anastomosis in an MR environment in which both vascular lumens can be monitored during deployment. Finally, these types of anastomoses may create new difficulties not previously seen with procedures such as TIPS. This includes stability of the anastomoses, potential for stricture formation, and formation of pseudoaneurysms. All these concerns will have to be addressed in long-term longitudinal studies.

\section{CONCLUSIONS}

Transcaval punctures to the portalmesenteric venous system are feasible with MR imaging guidance. Using a combination of MR imaging and conventional fluoroscopy for guidance, we were able to successfully create a percutaneous shunt and a vascular anastomosis between the portal mesenteric venous system and the IVC.

\section{References}

1. Hermann RE, Henderson JM, Vogt DP, et al. Fifty years of surgery for portal hypertension at the Cleveland Clinic Foundation: lessons and prospects. Ann Surg 1995; 221:459-466.

2. Kim WR, Brown RS Jr, Terrault NA, et al. Burden of liver disease in the United States: summary of a workshop. Hepatology 2002; 36:227-242.

3. Henderson JM, Gilmore GT, Hooks MA, et al. Selective shunt in the management of variceal bleeding in the era of liver transplantation. Ann Surg 1992; 216:248-254.

4. Henderson JM, Nagle A, Curtas S, et al. Surgical shunts and TIPS for variceal decompression in the 1990s. Surgery 2000; 128:540-547.

5. Henderson JM. Selective shunts in the 1990s. Liver Transpl Surg 1997; 3:552-555.

6. Henderson JM. Portal hypertension and shunt surgery. Adv Surg 1993; 26: 233-257.

7. Jawaid Q, Saeed ZA, Di Bisceglie AM, et al. Biliary-venous fistula compli- 
cating transjugular intrahepatic portosystemic shunt presenting with recurrent bacteremia, jaundice, anemia and fever. Am J Transplant 2003; 3:16041607.

8. Arepally A, Karmarkar PV, Weiss C, et al. Magnetic resonance image-guided trans-septal puncture in a swine heart. J Magn Reson Imaging 2005; 21:463467.

9. Ocali O, Atalar E. Intravascular magnetic resonance imaging using a loopless catheter antenna. Magn Reson Med 1997; 37:112-118.

10. Atalar E, Kraitchman DL, Carkhuff B, et al. Catheter-tracking FOV MR fluoroscopy. Magn Reson Med 1998; 40: 865-872.

11. Kaminou T, Rosch J, Yamada R, et al. Percutaneous retroperitoneal splenorenal shunt: an experimental study in swine. Radiology 1998; 206:799-802.

12. Vivas I, Bilbao JI, Martinez-Cuesta A, et al. Percutaneous extrahepatic portacaval shunt with covered prostheses: feasibility study. J Vasc Interv Radiol 2003; 14:1543-1552.

13. Wallace MJ, Ahrar K, Tinkey $\mathrm{P}$, et al. Transvenous extrahepatic portacaval shunt with use of a modified prototype stent-graft: experimental study in animals. J Vasc Interv Radiol 2005; 16:261267.

14. Wallace MJ, Ahrar K, Stephens LC, et al. Transvenous extrahepatic portacaval shunt: feasibility study in a Swine model. Radiology 2003; 228:119-125.

15. Kee ST, Rhee JS, Butts K, et al. 1999 Gary J Becker Young Investigator Award. MR-guided transjugular portosystemic shunt placement in a swine model. J Vasc Interv Radiol 1999; 10: 529-535.

16. Kee ST, Ganguly A, Daniel BL, et al. MR-guided transjugular intrahepatic portosystemic shunt creation with use of a hybrid radiography/MR system. J Vasc Interv Radiol 2005; 16:227-234.

17. Henderson JM. Role of distal splenorenal shunt for long-term management of variceal bleeding. World J Surg 1994; 18:205-210.

18. Petersen B, Uchida BT, Timmermans $\mathrm{H}$, et al. S-guided direct intrahepatic portacaval shunt with a PTFE-covered stent-graft: feasibility study in swine and initial clinical results. J Vasc Interv Radiol 2001; 12:475-486.

19. Niyyati M, Petersen BD, Pavcnik D, et al. A flexible stent with small intestinal submucosa covering for direct intrahepatic portocaval shunt: experimental pilot study in swine. Cardiovasc Intervent Radiol 2005; 28:215-220.

20. Seong CK, Pavcnik D, Uchida BT, et al. Experimental percutaneous extrahepatic portacaval shunt creation by transjugular approach in swine. Cardiovasc Intervent Radiol 2005; 28:616-623. 